\title{
A New Efficient Integrated Routing Scheme for SDH/SONET-WDM Multilayer Networks
}

\author{
Marc Necker and Christoph Gauger \\ Institute of Communication Networks and Computer Engineering \\ University of Stuttgart, Pfaffenwaldring 47, 70569 Stuttgart, Germany \\ \{necker,gauger\}@ind.uni-stuttgart.de
}

\author{
Stefan Bodamer ${ }^{1}$ \\ Marconi Communications Ondata $\mathrm{GmbH}$ \\ Stuttgarter Str. 139, 71522 Backnang, Germany \\ stefan.bodamer@marconi.com \\ Work was done while with the University of Stuttgart
}

\begin{abstract}
This paper presents and evaluates the new Weighted Integrated Routing scheme to solve the problem of dynamic routing and traffic grooming in meshed SDH/SONET-WDM multilayer networks. Its performance is compared to non-integrated schemes using a cost model.
\end{abstract}

\section{Introduction}

In optical networks, transmission rates have dramatically increased during the past years and dynamic provisioning of lightpaths will soon be available through an automated transport network control plane, e. g. ASTN [1]. Advanced node architectures which integrate electrical and optical layers will most likely be available in future systems. Assigning low-bandwidth electrical connections to high-speed optical lightpaths, also known as traffic grooming, is an important aspect in such networks, since small granularities are the major source of revenue for operators and provide service flexibility.

Routing on the electrical and optical layer is an essential component of every grooming strategy. Efficient transport of dynamic traffic demands of different granularities from the SONET/SDH hierarchy requires optimized multi layer routing and grooming algorithms. Non-integrated routing schemes treat both layers separately while integrated schemes try to improve performance by combining both layers. Most approaches for integrated routing, e. g. [2] (SONET) or [3] (IP), take advantage of complete knowledge about topology and occupancy of both layers which is not supported by the overlay network model [4]. However, the overlay model is favored from an operator point of view as information on transport networks is very sensitive and should be kept secret.

In this paper, we present Weighted Integrated Routing which solves the dynamic grooming problem without the need of gathering full state information of both layers. In addition, we introduce a method for dimensioning the number of transponders in multilayer nodes.

\section{Multilayer Grooming and Routing Options}

The SDH/SONET-WDM-network approach leads to a multilayer node model as shown in Fig. 1 left. This model comprises an optical cross-connect (OXC) on the optical layer, which is assumed to be non-blocking. The electrical layer mainly consists of a non-blocking electronic cross-connect (EXC), which is able to switch electrical connections at an arbitrary granularity. The EXC thus allows for an effective grooming of electrical connections. The EXC and OXC are connected by $z$ transponders and receivers.

In a centralized routing scheme, the routing control center has to choose a path within the network for each connection request. Four basic grooming options can be identified:

A single-hop grooming on existing lightpath:

The connection is assigned to one existing direct lightpath.

B multi-hop grooming on existing lightpaths:

Routing takes place on the electrical layer by using more than one existing lightpath and switching the connection in the EXCs of intermediate nodes.

C single-hop grooming on new lightpath:

A new lightpath is set up between the source and the destination node. The connection request is routed on the optical layer via this new lightpath. 


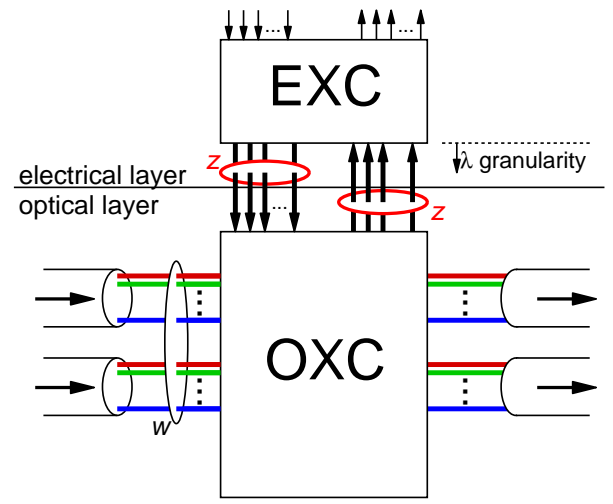

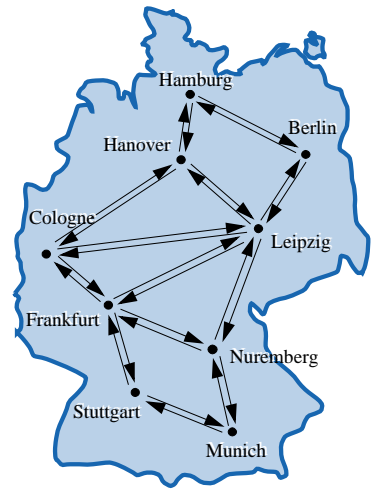

Figure 1. Multi-layer node and network topology

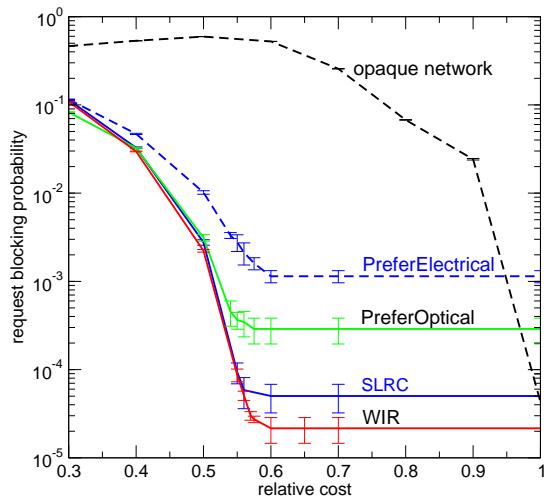

Figure 2. Request blocking probability

\section{D combined multi-hop grooming on new and existing lightpaths:}

This is a combination of options $\mathrm{A}$ and $\mathrm{C}$. The connection request can be routed on both the electrical and optical layer by using a series of existing and new lightpaths.

Non-integrated routing schemes are capable of grooming on either existing or new lightpaths. Only with integrated routing does the routing control center have enough information to also perform the combined grooming described in D. As a reference we will consider two different non-integrated routing schemes, namely PreferOptical and PreferElectrical (TLRC in [2]), which differ in the order they apply the different grooming policies until one succeeds. In PreferOptical, the options are applied in the order A-C-B, where for PreferElectrical the order is A-B-C.

\section{$3 \quad$ Weighted Integrated Routing (WIR)}

In contrast to the non-integrated routing schemes, WIR applies the different grooming strategies in parallel, including combined grooming. For a connection request, each possible path that results from a grooming strategy is rated according to a set of criteria. Finally, the path with the best rating is chosen.

Finding paths with combined grooming is harder than applying grooming purely on the electrical or optical layer. In [2], Zhu and Mukherjee use a reachability graph capturing all possible links for a given connection request between any two nodes for their proposed SLRC scheme. In our approach, we search combined grooming paths along pre-calculated routes, for which we used the first $N_{\text {MaxAlt }}$ shortest completely link disjoint paths from source to destination.

A route consisting of $N$ hops has $N-1$ intermediate nodes. The algorithm starts by selecting one intermediate node and rating all four possiblities for combined grooming with respect to that node (new-new, new-existing, existing-new, existing-existing). In this intermediate node, the connection traverses the EXC whereas it is kept on the optical layer in all other intermediate nodes. Afterwards, the remaining intermediate nodes are selected to gather rating information. If $N>2$, the algorithm additionally selects two intermediate nodes and rates all eight possibilites for combined grooming (new-new-new, new-new-existing, ...), eventually looking into all possibilities to select two intermediate nodes. Altogether, the search for combined grooming paths comprises all combinations of selecting up to $N_{\text {MaxVias }}$ intermediate nodes. Note that the routing control center only needs rating information from each individual layer. Non-existing or blocked lightpath requests can be signaled with an infinitely high cost.

In order to rate the different paths, rating functions are introduced which represent the cost of the paths. Two functions are needed to rate single lightpaths and paths consisting of more than one lightpath:

$$
\begin{gathered}
C_{\text {lightpath }}=\left(\frac{N}{N_{\text {min }}}-1\right) \cdot W_{\text {OptHops }} \\
C=N_{\text {Vias }} \cdot W_{\text {transponder }}+W_{\text {lighpath }} \cdot \max _{N_{\text {Vias }}+1}\left(C_{\text {lighpath }_{m}}\right) .
\end{gathered}
$$



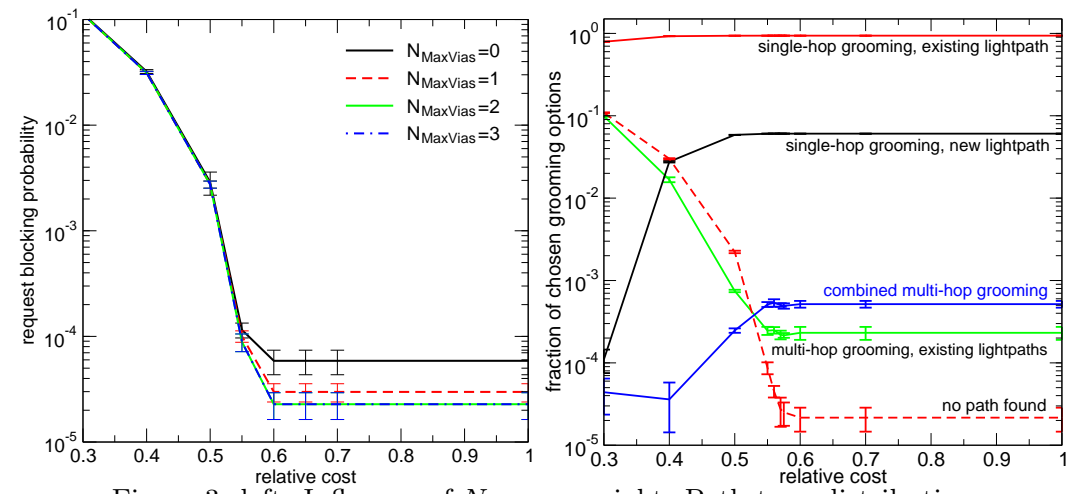

Figure 3. left: Influence of $N_{\text {MaxVias }}$, right: Path type distribution

$N_{\text {Vias }}$ is the number of intermediate in which where the connection traverses the EXC and transponders. $N_{\min }$ is the number of hops of the shortest possible lightpath connecting the end nodes of the lightpath being rated. All weighting factors $W$ have a default value of 1 .

In contrast to other integrated routing schemes which require complete information of the electrical as well as the optical layer [2,3], WIR probes rating information on different paths from both layers and selects a path based on the cost $C$. This is a significant advantage for network operators, that often want to hide network details from customers.

\section{Simulation Studies}

All presented simulation studies were performed using a fictitious 9-node network of Germany, the topology of which is shown in Fig. 1 right. This network was introduced and dimensioned for static traffic demands [5] such that links contain a certain number of fibers each holding 8 wavelengths. The ratio of offered traffic load in the dynamic scenario and traffic load used for static dimensioning defines the system load chosen to be $70 \%$ for all presented studies. On the optical layer, the dynamic routing scheme ADR3 [5] is applied, while the electrical layer uses a Dijkstra algorithm to compute the shortest route online.

For a multilayer node, the number $z$ of transponders is a crucial parameter, from a performance as well as a cost point of view. Particularly, for a given network topology, transponders are the major variable cost factor. Hence, we define the absolute cost as the sum of all transponders in the network. We used a model based on the well-known Erlang-B formula to calculate $z_{k}$ for each node $k$ from the traffic offered at that node with $z_{k} \leq w_{k}$ as a boundary condition. Given a specific absolute cost and the offered traffic at each node, $z_{k}$ can uniquely be determined for any node. For convenience, the relative cost is introduced as the absolute cost normalized by the sum of all $w_{k}$ at all nodes $k$. It is advantageous to compare different routing schemes based on the absolute or relative cost.

A traffic mix consisting of $80 \%$ STM-1, $15 \%$ Gigabit-Ethernet (transported as VC-4-7v in SDH [6], but without diverse routing) and 5\% STM-16 connection requests was used. The bandwidth of a wavelength was chosen to be STM-16. Connection requests arrive according to a Poisson Process with exponential holding times.

Fig. 2 shows the blocking probability for different schemes. As a reference, the graph contains the performance of an opaque network with static point-to-point lighpath connections of adjacent nodes, which is only comptetitive at a relative cost of almost $100 \%$. The non-integrated routing scheme PreferOptical outperforms the scheme PreferElectrical by almost one order of magnitude. The integrated WIR achieves a request blocking probability, which is about one order of magnitude below the best non-integrated scheme and slightly below SLRC. There is a noticeable bend in all curves at a cost of about 0.55. For lower costs, the request probability is dominated by blocking at the transponders. For larger costs, there are sufficient transponders available so that wavelength blocking dominates. 
Fig. 3 left shows the request blocking probability for different values of $N_{\text {MaxVias. For }} N_{\text {MaxVias }}=0$, there is no possibility for combined grooming. Still, WIR outperforms the non-integrated schemes due to the use of rating functions. Increasing $N_{\text {MaxVias }}$ by one, which means including combined grooming at the lowest level, lowers the request blocking probability by half an order of magnitude. Further increasing $N_{\text {MaxVias }}$ gives diminishing returns. For all $N_{\text {MaxVias }} \geq 2$ the results are identical due to the limited path length in the Germany network. Not shown are the results when varying $N_{\text {MaxAlt }}$, since there is only a negligible difference in performance.

Fig. 3 right shows that for the relevant cost range combined grooming and multi-hop grooming on existing lightpaths are only used for approximately $0.1 \%$ of all connection requests. However, as was seen in Fig. 3 left, the relatively small portion of combined grooming paths already has a significant impact on system performance.

\section{Conclusion}

In this paper we presented a new integrated multilayer scheme with reasonable complexity that outperforms non-integrated and even more complex integrated schemes. Improved performance is achieved even without the need for a full integrated view of the network.

\section{References}

1. ITU-T G.astn Draft V.0.3, "Architecture for the Automatic Switched Transport Network (ASTN)"

2. K. Zhu, B. Mukherjee: "On-Line Approaches for Provisioning Connections of Different Bandwidth Granularities in WDM Mesh Networks", OFC 2002, Anaheim

3. M. Kodialam, T.V. Lakshman: "Integrated Dynamic IP and Wavelength Routing in IP over WDM Networks", INFOCOM 2001, New York City, Vol. 1, pp. 358-366

4. E. Modiano: "Traffic Grooming in WDM Networks", IEEE Communications Magazine, Vol. 39, No. 7, 2001, pp. 124-129

5. J. Späth: "Dynamic routing and resource allocation in WDM transport networks", Computer Networks, Vol. 32, No. 5, 2000, pp. 519-538

6. ITU-T Rec. G.707/clause 11, "Network Node Interface for the Synchronous Digital Hierarchy (SDH)", 2000 